\title{
Preliminary Numerical Assessment of Turbulent Flow Control with Plasma Actuators
}

\author{
A. Criscione ${ }^{1}$, B. Frohnapfel ${ }^{2}$ and C. Tropea ${ }^{1,2}$ \\ 1 Fachgebiet Strömungslehre und Aerodynamik, \\ 2 Center of Smart Interfaces, Technische Universität Darmstadt, Germany \\ a.criscionedsla.tu-darmstadt.de
}

\begin{abstract}
Summary
A numerical investigation of damping of near-wall spanwise velocity fluctuations in a turbulent channel flow is carried out. Spanwise damping is realized with a body force which mimics the characteristic force distribution of plasma actuators. This force distribution is implemented in a direct numerical simulation. The body force is triggered by the signal of a sensor in upstream location. The control loop is applied with different actuator configurations, considering a spatially continuous and discontinuous body force distribution at the wall. The influence of distributed sensors and actuators of finite size on the control loop performance is investigated. The results show a reduction of the skin friction at the wall with maximum power saving rates up to $20 \%$.
\end{abstract}

\section{Introduction}

The control of turbulent flows is a key issue in many engineering applications since it is related to power savings, efficient production processes and possible solutions for global environmental problems. In this regard, the plasma actuator is an attractive actuator device due to its low mass and a rather simple system integration. Flow control applications of plasma actuators include separation control [8], transition control [3] and wake control [6].

We investigate whether it might be possible to also use plasma actuators for skin friction drag reduction in turbulent flows. In the literature, attempts of imitating spanwise wall oscillation or related predetermined active control schemes [7] with plasma actuators have been reported [10]. We also consider the introduction of the plasma actuator body force in the spanwise direction $\left(x_{3}\right)$, but in contrast to the approaches in the field of predetermined control, we focus on reactive control schemes with the goal to damp instantaneous spanwise velocity fluctuations, $u_{3},[4,5]$ in the near-wall region.

We carry out direct numerical simulation of a fully developed channel flow $\left(R e_{\tau}=150\right)$ where the near-wall body force is introduced as an additional term on the right hand side of the Navier-Stokes equations. The distribution of this body force is chosen to mimic a characteristic force distribution of the plasma actuator. Throughout this paper the superscript ()$^{+}$indicates 
normalization with the kinematic viscosity, $\nu$, and the wall shear velocity, $u_{\tau}$, of the uncontrolled channel flow.

\section{Body Force Introduced by a Plasma Actuator}

The Single Dielectric Barrier Discharge (SDBD) actuator, generally referred to as plasma actuator, consists of two electrodes placed parallel to each other. The lower electrode is covered with an insulating film, the so-called dielectric barrier, while the upper electrode is exposed to the flow. This set-up is known to produce a body force in the fluid above the actuator when a high AC voltage is applied to the electrodes. The physics of the generation of this body force as well as its exact distributions in time and space are still a subject of investigation; see e.g. [1,6] for further details and references.

For our numerical experiment we consider only the time averaged force distribution of a plasma actuator since the characteristic frequencies of the force modulation are significantly faster than the highest frequencies in the turbulent flow field [6]. In the literature different models are available, which describe the distribution of this net body force introduced by a plasma actuator. We consider two models with complementary approaches. First, an empirical model in which the force distribution above the actuator is extracted from experimental velocity field data [9]. Second, an electrochemical model for which the space and time evolution of the charged particle densities, the electrical field and the surface charges are computed numerically [1]. The distribution of the wall-parallel force component obtained with these models is plotted in figure 1. Both models also provide a wall-normal component of the body force. However, this component has a much smaller integral value and its distribution significantly differs for different models.

Although the physical dimensions of the considered actuators are significantly different, a common distribution at the location of the maximum body force can be extracted. The wall normal distribution at this location is characterized by a strong increase of the body-force in the wall vicinity, which is followed by an almost exponential decay with increasing wall distance. In order to be able to implement a body force distribution in our numerical code which represents a plasma actuator we describe this behavior with a fitting function such that $b f_{3}^{+} \propto x_{2}^{+} e^{-x_{2}^{+}}$.

\section{The Control Loop}

In order to damp the spanwise velocity fluctuations, $u_{3}$, in the near-wall region of a turbulent channel flow their direction and magnitude needs to be known. However, their direct measurement cannot be realized in practice. Lee and Kim [5] showed that the spanwise wall shear stress, $\tau_{w, x_{3}}$, at the actuation location can be successfully used as sensor input for a control scheme that 

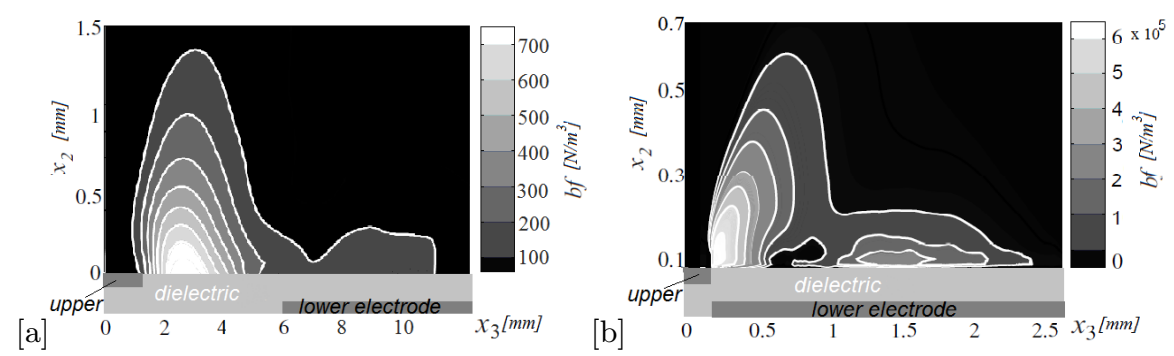

Figure 1 Body force distribution of the empirical [a] (dielectric thickness $=1.5$ $\mathrm{mm}$ ) and of the electrochemical model [b] (dielectric thickness $=0.5 \mathrm{~mm}$ )

damps $u_{3}$. However, it should be noted that in this case the near-wall region in which $u_{3}$-damping can be realized is limited to $x_{2}^{+} \approx 10$, because the correlation between $\tau_{w, x_{3}}$ and $u_{3}\left(x_{2}\right)$ quickly deteriorates outside this region.

Since a measurement of $\tau_{w, x_{3}}$ at the actuation location is difficult to realize, Frohnapfel et al. [4] suggested to use the spanwise gradient of the streamwise wall shear stress, $\partial \tau_{w, x_{1}} / \partial x_{3}$, upstream of the actuator location as sensor input. We follow that line and formulate our control loop in such a way that the plasma actuator body force is introduced into the numerical simulation as:

$$
b f_{3}^{+}=\frac{1}{\Phi^{+}} x_{2}^{+} e^{-x_{2}^{+}} \cdot \overbrace{\frac{\partial \tau_{w, x_{1}}}{\partial x_{3}}\left(x_{1}^{+}-\Delta x_{1}^{+}\right)}^{\text {upstream sensing }} f\left(x_{2}^{+}\right) .
$$

In this formulation $\left(\Phi^{+}\right)^{-1}$ is the relaxation time constant which, in combination with the sensor value $\frac{\partial \tau_{w, x_{1}}}{\partial x_{3}}\left(x_{1}^{+}-\Delta x_{1}^{+}\right)$, determines the strength of the body force; $f\left(x_{2}^{+}\right)$is a step function which limits the wall-normal extension of the region where the body force is applied to ten viscous units on the top and bottom wall. We refer to this layer as damping layer, $y_{d}$, such that $f\left(x_{2}^{+} \leq y_{d}^{+}\right)=1$ and $f\left(x_{2}^{+}>y_{d}^{+}\right)=0$. According to the results of previous work [4] we set the distance between sensor and actuator to $\Delta x_{1}^{+}=50$. Figure 2 shows the wall-normal distribution of the time averaged body force for different $\left(\Phi^{+}\right)^{-1}$.

For active flow control methods, the control performance can be captured with different indicators [2]. For a simulation with constant bulk velocity, the pumping power, $P$, required to drive the flow, is directly proportional to the average streamwise wall shear stress, $\overline{\tau_{w, x_{1}}}$, and thus a measure for the obtained drag reduction. The additional power input per unit wall area, $P_{i n}$, due to the applied control can be obtained by including the body force into the energy equation of the flow field. For the present case it is given by:

$$
P_{\text {in }}=\int_{0}^{H}|\overbrace{b f_{3} \cdot u_{3}}^{P_{3}}| d x_{2}
$$


where $H$ corresponds to the channel height and the overbar indicates averaging in time as well as in the streamwise and spanwise directions. The local power input, $P_{3}$, assumes a negative value if the body force indeed damps the spanwise velocity fluctuations. Positive values of $P_{3}$ indicate that the control loop does not work as anticipated and the body force enhances the existing velocity fluctuations.

For active flow control the drag reduction rate, $R$, should be replaced by the power saving rate, $S$, which takes into account the additional power consumption, $P_{i n}$, of the actuator. Another important performance indicator is the gain, $G$, which indicates how much power is saved per control power input:

$$
S=\frac{P_{0}-\left(P+P_{i n}\right)}{P_{0}}=R-\frac{P_{i n}}{P_{0}}, \quad G=\frac{P_{0}-P}{P_{i n}} .
$$

$P_{0}$ refers to the power consumption of the uncontrolled flow. In order to obtain the most conservative values for $S$ and $G$ we modify the formulation of $P_{i n}$ such that the absolute value of $b f_{3} \cdot u_{3}$ is taken before the spatial and temporal averaging is applied $\left(\left|\overline{b f_{3} \cdot u_{3} \mid}>\right| \overline{b f_{3} \cdot u_{3}} \mid\right)$. Since we have defined $P_{\text {in }}$ in such a way that all possible energy losses associated with actuators, sensors, control circuits and so forth are neglected, $G$ determines the lower bound of the system efficiency $\eta$ required to obtain net power savings: $\eta>G^{-1}$.

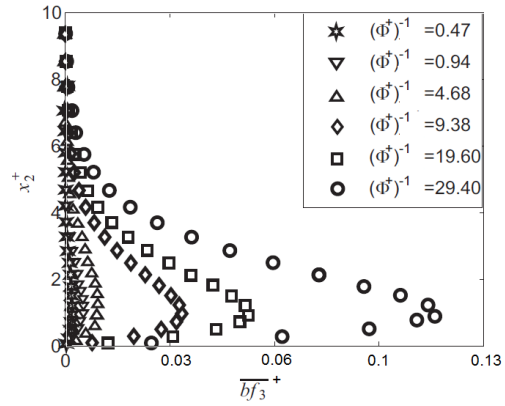

Figure 2 Body force distribution plotted for different $\left(\Phi^{+}\right)^{-1}$

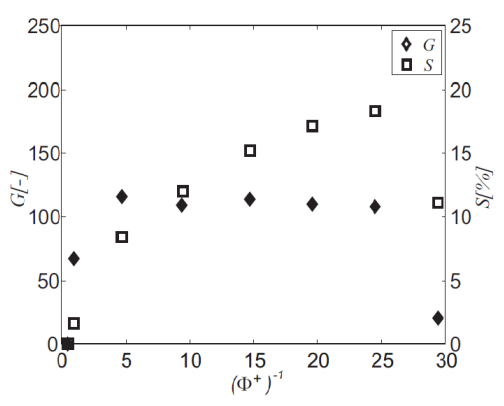

Figure 3 Effect of the forcing strength $\left(\Phi^{+}\right)^{-1}$ on $\mathrm{S}$ and $\mathrm{G}$

\subsection{Spatially Continuous Control}

Most numerical flow control research is carried out with spatially continuous control where the control input is realized at every grid point assuming infinitely small actuators that are continuously distributed along the wall. In order to enable comparison with other control schemes we will also consider this case before considering the effect of finite size sensors and actuators in 
the next section. Figure 3 shows the results of the applied control in terms of $\mathrm{G}$ and $\mathrm{S}$. It can be seen that the power saving rate, $S$, increases with increasing forcing strength up to almost $20 \%$ before it starts to decrease. The gain, $G$, remains almost constant for a broad range of $\left(\Phi^{+}\right)^{-1}$-values before it decreases at the same forcing strength where reduced values of $S$ are registered. This trend can be explained when considering the local power input, $P_{3}$, as given in equation 2 .

$P_{3}$ is negative if the body force acts as a damping force and positive if it acts as a $u_{3}$-enhancing force. Figure 4 depicts the local power input due to the applied body force as a function of wall distance. For small forcing strengths, i.e. small $\left(\Phi^{+}\right)^{-1}$, the body force acts as a damping force in the entire damping layer. For stronger forcing, positive values of $P_{3}$ can be found in the vicinity of the wall while $P_{3}$ is negative in the outer region of the damping layer. This indicates that the distribution and intensity of $b f_{3}$ is such that spanwise velocity fluctuations in the vicinity of the wall are not only damped but forced to change their sign. For a further increase of the forcing strength this area of fluctuation enhancement increases up to the point where numerical instabilities occur. This upper limit for the strength of the applied body force arises due to the employed feed-forward control loop. For weak control input the spatial correlations between $\frac{\partial \tau_{w, x_{1}}}{\partial x_{3}}\left(x_{1}^{+}-\Delta x_{1}^{+}\right)$and $-\tau_{w, x_{1}}$ on which the control is based, is not modified significantly. However, for stronger body forces the correlation does not exist any more and thus the control loop breaks down. Considering the distribution of $P_{3}$ in figure 4 and

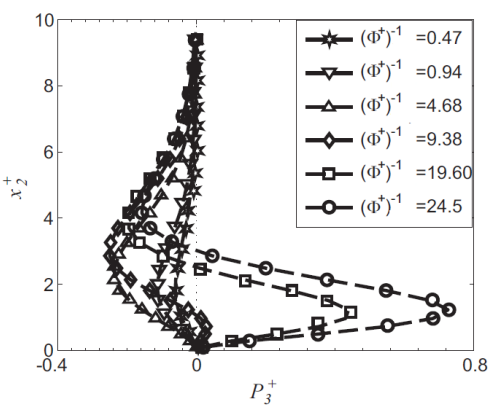

Figure 4 Local energy input, $P_{3}^{+}$, plotted against the strength factor $\left(\Phi^{+}\right)^{-1}$

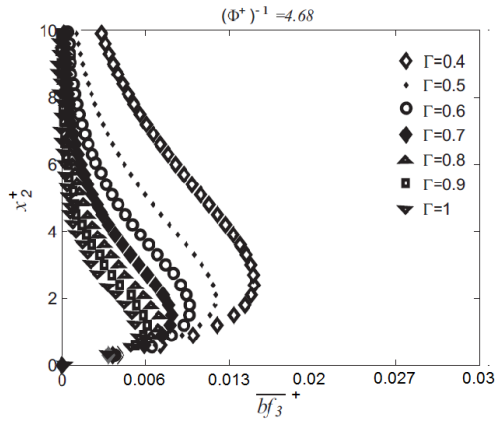

Figure 5 Body force distribution for different values of the sliding factor $\Gamma$

the distribution of $b f_{3}$ in figure 2 , it is obvious that the peak value of $b f_{3}$ in the vicinity of the wall causes the first occurrence of positive values for $P_{3}$. In order to realize higher power saving and gain rates it might therefore be desirable to shift the location of the maximum body force further away from 
the wall. A simple shift of the location of the maximum body force can be obtained by modifying the formulation of the body force as follows:

$$
b f_{3}^{+}=\frac{1}{\Phi^{+}}\left(x_{2}^{+}\right) e^{-\Gamma x_{2}^{+}} \cdot \overbrace{\frac{\partial \tau_{w, x_{1}}}{\partial x_{3}}\left(x_{1}^{+}-\Delta x_{1}^{+}\right)}^{\text {upstream sensing }} f\left(x_{2}^{+}\right) .
$$

If the sliding factor $\Gamma$ is set to values smaller than one, the barycenter of the body force will move further away from the wall. This upward shift of the maximum location will also yield an increased integral value of $b f_{3}$ such that the strength of the applied force is no longer given by $\left(\Phi^{+}\right)^{-1}$ alone, but by a combination of $\Gamma$ and $\Phi$. Nevertheless, by varying $\Gamma$ we are able to obtain the information whether higher values of $S$ and $G$ can in general be obtained with a different force distribution. The influence of varying $\Gamma$ on the distribution of $b f_{3}$ for a fixed value of $\left(\Phi^{+}\right)^{-1}$ is shown in figure 5 .

Figure 6 shows the effect of varying the sliding factor, $\Gamma$, for $\left(\Phi^{+}\right)^{-1}=$ 4.69 on $S$ and $G$. The maximum power saving rate, $S$, differs only slightly from the maximum value obtained for $\Gamma=1 \mathrm{in}$ fig. 3 . However, a significant increase of $G$ with a maximum value above 200 for $\Gamma=0.4$ is obtained. This result indicates that an optimization of the force distribution for damping of the spanwise velocity component within $y_{d}^{+}=10$ is possible.

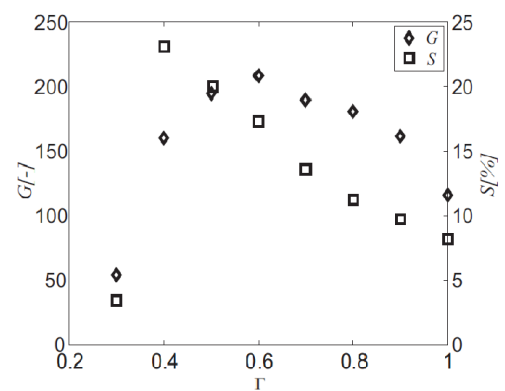

Figure 6 Effect of $\Gamma$ on $\mathrm{S}$ and $\mathrm{G}$ for the fitting function model at $\left(\Phi^{+}\right)=4.69$

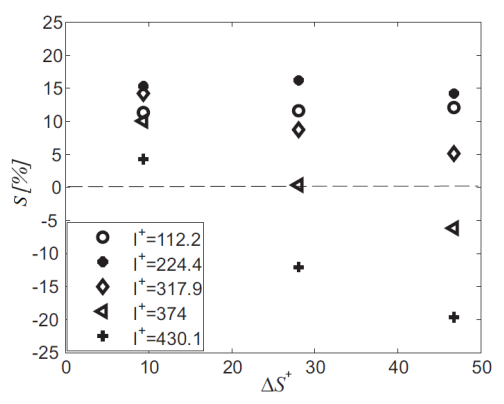

Figure $7 \quad \mathrm{R}$ over $\Delta S^{+}$as a function of the actuator length, $l^{+}$, for $d x^{+}=561$

\subsection{The Influence of Finite Size Actuators}

For considerations towards a realistic control scheme, the finite actuator size needs to be taken into account. Figure 8 shows an exemplary control configuration at the wall of the channel with defined placement parameters: $l^{+}$ and $h^{+}$are the length and width of the plasma actuator, respectively, $d x^{+}$ 
and $d z^{+}$refer to the streamwise and spanwise distance between the actuators, while $\Delta S^{+}$defines the distance between sensor and upstream corner of the actuator. In a parameter study we find that the actuator can easily be extended in the streamwise direction up to approximately 300 viscous units without significant influence on the power saving rate. However, the extension in spanwise direction is rather critical and doubling the actuator width from $h^{+}=7.5$ to $h^{+}=15$ resulted in negative power saving rates.

An exemplary result of the parameter study is shown in fig. 7 . In this case the distance between two consecutive actuators, $d x^{+}$, is chosen such that only two actuators can be placed in the streamwise direction of the computational domain. These two actuators are varied in streamwise length, $l^{+}$, and also the placement of the sensor, $\Delta S^{+}$is modified. It can be seen that actuator lengths well above 100 viscous units can be realized without significantly decreasing the resulting power saving rate. The extension of the actuator in streamwise direction influences the optimum placement of the sensing device which should be moved closer to the actuator for increasing $l^{+}$.

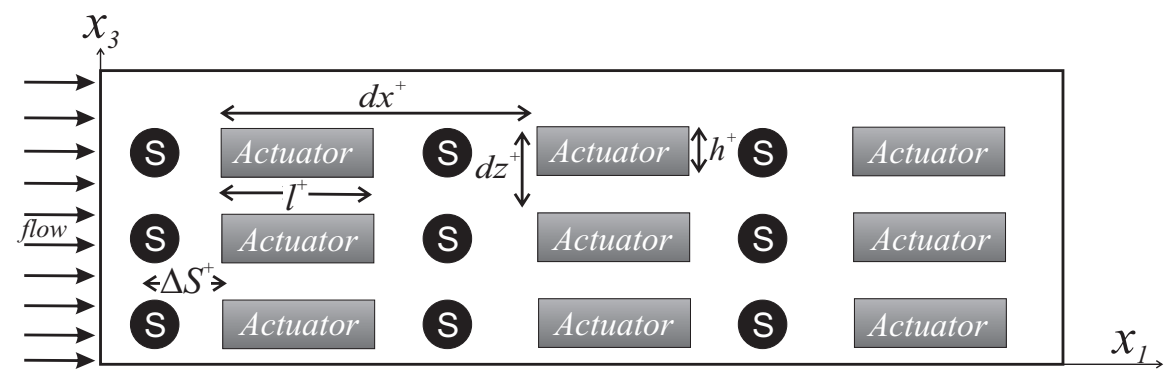

Figure 8 Discontinuous control configuration

\section{Conclusion}

In order to obtain information about whether or not plasma actuators might be suitable devices for the application in reactive control schemes for turbulent skin friction drag reduction a numerical experiment is carried out. The force distribution of two fundamentally different models of the plasma actuator are compared in order to extract a characteristic distribution which can be implemented in the numerical simulation. In the numerical experiment we employ a feed-forward control loop in which the spanwise gradient of the streamwise wall shear stress upstream of the actuator location serves as sensor input.

We find that the force distribution extracted from the plasma actuator models can be successfully used in the employed reactive control loop yielding 
power saving rates of more than $20 \%$. It is shown that an improvement of the control loop performance can be obtained if the barycenter of the body force is moved away from the vicinity of the wall.

In respect to the spatial extension of a plasma actuator it is found that the present feed-forward control loop can be run with actuators that have a streamwise length of up to 300 viscous units. However, an extension of the actuator in the spanwise direction seems to be critical. According to the present results, the spanwise extent of the region in which the body force is triggered by the same sensor signal should be limited to less than ten viscous units, which roughly corresponds to half the radius of a quasi-streamwise vortex. Finally, it should be noted that a number of additional issues, including the limits in miniaturizing the plasma actuator, the influence of the wall normal force component that we have neglected so far and also the influence of sensor and actuator response times, need to be addressed before a final statement about the possible use of plasma actuators in reactive turbulent flow control loops can be made.

Acknowledgements. The authors would like to thank N. Kasagi, Y. Hasegawa (The University of Tokyo) and J. Kriegseis (TU Darmstadt) for valuable discussions and M. Yokoo (The University of Tokyo), without his solver CHFLOW our work would not have been possible.

\section{References}

[1] Boeuf, J.P., Langmich, Y., Unfer, T., Callegari, T., and Pitchford, L.C. : Electrohydrodynamic force in dielectric barrier discharge plasma actuators. Journal of Physics D: Applied Physics (2007) 40 652-662

[2] Frohnapfel, B., Hasegawa, Y., Kasagi, N., and Grosse, S. : Estimation of the spanwise wall shear stress based on upstream information for wall turbulence control. Proc. 12th Euromech European Turbulence Conference (2009), 209-212.

[3] Grundmann, S. : Transition control using dielectric barrier discharge actuators. Shaker Verlag (2008)

[4] Kasagi, N., Hasegawa, Y., and Fukagata, K. : Toward cost-effective control of wall turbulence for skin friction reduction. Advances in Turbulence XII (2009) $189-200$

[5] Lee, C. and Kim, J. : Control of the viscous sublayer for drag reduction. Physics of Fluids (2002) 14 2523-2530

[6] Moreau, E. : Airflow control by non-thermal plasma actuators. Journal of Physics D: Applied Physics (2007) 40 605-636

[7] Quadrio, M. : Drag reduction in turbulent boundary layers by in-plane wall motion. Philosophical Transactions of the Royal Society A (2010) to appear

[8] Roupassov, D.V., Nikipelov, A.A., Nudnova, M.M., and Starikovskii, A.Y. : Flow separation control by plasma actuator with nanosecond pulsed-periodic discharge. AIAA Journal (2009) 47 168-185

[9] Wilke, B. : Aerodynamische Strömungssteurung mittels dielektrischen Barrierenentladungs-Plamaaktuatoren. Shaker Verlag (2009)

[10] Wilkinson, S.P. : Investigation of an oscillating surface plasma for turbulent drag reduction. AIAA 2003-1023 